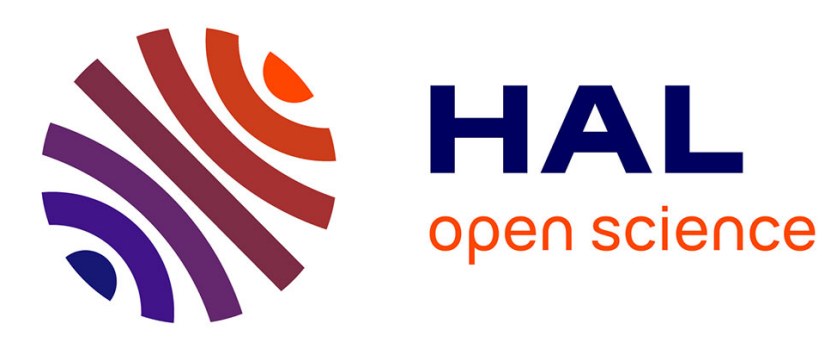

\title{
Holography in education and popular science: a new versatile and vibrationless color device
}

\author{
E. Salançon, E Escarguel
}

\section{To cite this version:}

E. Salançon, E Escarguel. Holography in education and popular science: a new versatile and vibrationless color device. European Journal of Physics, 2019, 40 (1), pp.015301. 10.1088/1361-6404/aae8ba . hal-01962540

\section{HAL Id: hal-01962540 \\ https://hal-amu.archives-ouvertes.fr/hal-01962540}

Submitted on 1 Feb 2019

HAL is a multi-disciplinary open access archive for the deposit and dissemination of scientific research documents, whether they are published or not. The documents may come from teaching and research institutions in France or abroad, or from public or private research centers.
L'archive ouverte pluridisciplinaire HAL, est destinée au dépôt et à la diffusion de documents scientifiques de niveau recherche, publiés ou non, émanant des établissements d'enseignement et de recherche français ou étrangers, des laboratoires publics ou privés.

\section{(c)(1)}

Distributed under a Creative Commons Attribution| 4.0 International License 


\title{
Holography in education and popular science: a new versatile and vibrationless color device
}

\author{
E. Salançon ${ }^{1}$ and A. Escarguel ${ }^{2}$ \\ ${ }^{1}$ CINaM, Aix-Marseille Univ, CNRS, UMR7325, campus de Luminy-case 913, Marseille, 13009, \\ France \\ ${ }^{2}$ Laboratoire PIIM, Aix Marseille Univ, CNRS, UMR 7345, avenue escadrille Normandie-Niemen, \\ Marseille, 13013, France \\ E-mail: salancon@cinam.univ-mrs.fr
}

\begin{abstract}
Following the success of the pedagogic tool for monochromatic holography realized for pedagogic and popular science purposes a few years ago [Th. Voslion and A. Escarguel, Eur. J. Phys 33, 1803 (2012)], an upgrade has been developed. The resulting device includes all the necessary equipment to produce far more spectacular 4" $\times 5$ " color holograms for science outreach purposes. Graduate schools are also offered several experiments such as holographic diffraction gratings. Optimized to be extremely robust to vibrations, particularly by using the weight of the object to avoid unwanted vibrations during hologram exposure, the robustness of this device is demonstrated through optical microscopy measurements.
\end{abstract}

Keywords: Optical holography, physics education, popular science

PACS: $01.40 . \mathrm{Fk} ;$ 42.40.Eq; 42.40.Pa; 42.40.H t

\section{Introduction}

The reproduction and capture of the image has always been a fascinating challenge. Humans began by painting. Then photography was invented in the $19^{\text {th }}$ century, and constantly improved. The latest phase of this development is being able to capture the relief of a real scene.

Holography has a particular place in this history, as it is the only way to record the "whole" relief of a scene [1]. While other processes record the 3D effect for one or a few points of view, holography records a huge set of points of view of the scene, some holograms offering a $3 \mathrm{D}$ effect over $360^{\circ}$. This is due to the fact that interference is a non-local effect. Each point of the object illuminated by the coherent wave diffuses the information over a wide part of the photosensitive plate, giving rise to an interference pattern containing the 3D information. A hologram has high redundancy of information, which is why it is possible to see the whole object using only one part of the plate.

Producing a hologram requires at least one bright light source whose coherent beam is divided into two parts, the object and the reference beams. The first called the object beam is directed onto the object and retains a footprint of the wavefront deformation induced by its relief. The second, or reference beam, is directed onto the recording medium. Its 
interference with the object beam is recorded to reveal the phase deformation induced by the object, and thus its relief. For light-diffusing objects, this information is recorded over the whole hologram [2].

Anyone looking at a hologram thinks that holography is a "kind of magic", even though the underlying physics of interference of coherent light is today well understood. This makes holography a very motivating tool for engaging students and teaching them fundamental/applied optics [3,4]. For popular science purposes, realizing a live hologram has a strong impact on the audience, opening the way to discussions and thereby to sharing the knowledge coming out of research laboratories with the public.

This paper details a holography color device that is an upgrade of the pedagogic tool for monochromatic holography developed a few years ago [5,6]. The addition of a second, green laser mixed with the red laser allows color holograms to be realized, affording new experiments for university students and continuing education [7].

Because it is light and easy to move, this new holography color device is very practical for use in amphitheater courses demonstrations or for science outreach purposes. It can be used just as effectively in outdoor popular science events as in high level physics teaching. Since the degree of noise will differ greatly according to the situation, this upgraded device is fitted with two different plate-object supports, one for noisy conditions and the other for quieter conditions. The success of the first support under noisy conditions is largely guaranteed by the same principle as in the previous pedagogic tool [5]: the good horizontal contact between the plate and the glass ensures less vibrations. This last point is verified and the effectiveness of the support is tested by measuring spatial resolution and interferometric visibility on the recording plates for different noisy environments. Finally, we illustrate how this holography color device is used through two examples: color Denisyuk holograms and holography diffraction gratings.

\section{Description of the holography color device}

The holography color device is a $60 \mathrm{~cm} \times 40 \mathrm{~cm} \times 30 \mathrm{~cm}$ case containing everything required to produce color holograms under any noise conditions. The case itself is used as a dualbeam diverging light source during exposure. The device has been optimized to be simple, robust and to allow easy optical alignment when necessary.

To ensure good mixing of the two laser beams, the main component of the device is an optical setup fixed on a small $(400 \times 400 \mathrm{~mm})$ breadboard with three Sorbothane isolating feet fixed to the bottom of the case (figure 1). Sorbothane was chosen because its high shock attenuation [8] keeps the dual beam stable during exposure. The setup consists of a $20 \mathrm{~mW}-632.8 \mathrm{~nm}$ HeNe laser, a $20 \mathrm{~mW}-532 \mathrm{~nm}$ DPSS laser (class IIIb), a dichroic mirror to merge the collimated beams, a rotating polarizing filter in front of the green beam to control the red/green intensity ratio, and a glass convex lens placed in the path of the two mixed beams,
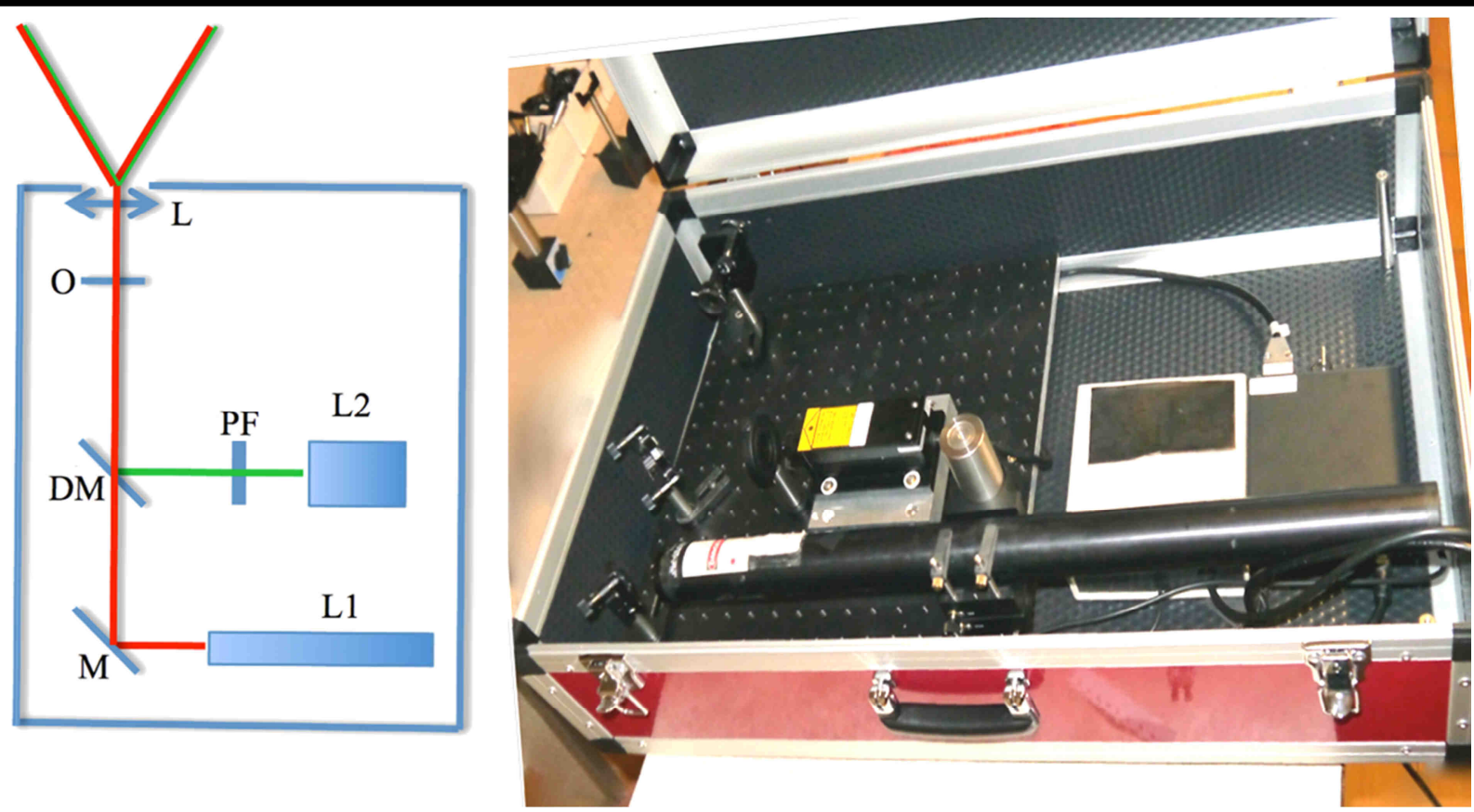

Figure 1: Diagram of the holography color device with the optical setup inside a case (left) and photograph (right). L1: 20mW 632.8nm HeNe laser (right fig.: black cylinder); L2: 20mW 532nm DPSS laser (right fig.: black rectangle); PF: polarizing filter; DM: dichroic mirror; M: front surface mirror; O: shutter; L: lens. In the photograph, the exit port and the lens L are located on the upper left side of the case. A small case contains the supplementary material and is placed on top of the laser power supply. 
just in front of an exit hole perforated in the case. The front surface mirror and the dichroic mirror are fixed on kinematic mounts with manual adjusters for easy optical alignment. A zero aperture iris is placed just in front of the lens, making it possible to block the laser beams. The short convex lens $(\mathrm{f}=20 \mathrm{~mm})$ reduces laser hazard: only diverging beams are accessible outside the case.

The two laser beams are mixed via the dichroic mirror, expanded via the lens, and horizontally reach one of the two supports described below (figure 2).
To upgrade the monochromatic pedagogic tool, the PO1 support was designed to produce contact reflection holograms, also called Denisyuk holograms [9], under noisy conditions. It is optimized for $4 " \times 5 "$ plates (figure 2 ): the reflecting prism has been replaced by a cheaper and larger front surface mirror to reflect the laser beams onto a large part of the holographic plate. For better stability, the photosensitive holographic plate is mounted on three columns with balls at their ends. Beams are deviated vertically by the $45^{\circ}$ mirror, crossing the transparent

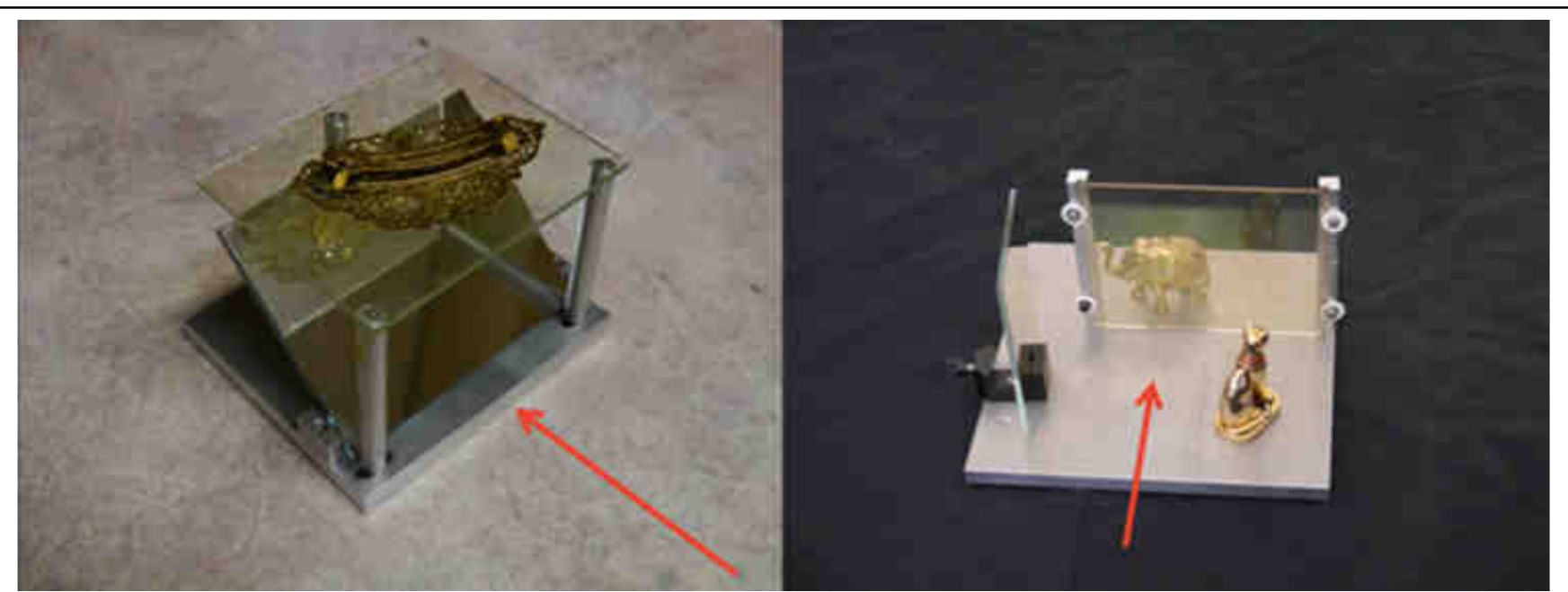

Figure 2: Plate/object supports PO1 (left) and PO2 (right); the arrows indicate the orientation of the diverging laser beams. The plate/object supports are placed far enough from the lasers so that the maximum surface of the 4 " $\times 5$ " plates is exposed. PO2 is used for single-shot reflection / transmission holograms: a reflection hologram of the elephant and a transmission hologram of the cat are produced simultaneously.

The HeNe laser has a coherence length of about $150 \mathrm{~mm}$, while the green laser is monomode with a much greater coherence length. Thus, for Denisyuk single-beam color holograms, the maximum distance $D=75 \mathrm{~mm}$ between the holographic plate and the object is imposed by the red laser (the depth of the object is limited to $75 \mathrm{~mm}$ ). These lasers cost at least $3800 €$, too expensive for a commercial kit aimed at general public use. But this is what it costs to realize high quality holograms with $100 \%$ success for pedagogic/popular science purposes. Economies could be made by replacing HeNe/DPSS with cheaper laser diodes. However, it is well known that they are not stable enough in terms of frequency, leading to lower-quality holograms and a much lower success rate in their realization. Moreover, these laser diodes are extremely fragile, and particularly vulnerable to static electricity. This necessitates great care in handling them and reduces their lifetime.

Two plate/object supports are included in the case: the first support (PO1) is used in noisy environments, and the second (PO2) when there is less noise. During exposure, the chosen support is placed so that the object is a few meters away from the hole of the case. photosensitive plate and being back-diffused by the object which is simply placed on top of the plate. This takes advantage of gravity to avoid any relative movement during the realization of the hologram, thus enabling holograms to be created even in noisy environments like a university amphitheater crowded with students or a science outreach session with children. This robustness is due to the fact that in holography, it is only after separation of the laser beam into two parts, the reference and the object beams, that any micrometric vibrations need to be prevented [10]. In our case, as described in the previous article [5], this implies avoiding any vibration of the object with respect to the plate on which it is placed. As in the first pedagogic tool, the plate/object support is simply placed far enough from the case to ensure that the combined red/green diverging beams deviated by the $45^{\circ}$ mirror illuminate the largest surface of the photosensitive plate. Typical exposure times are a few seconds. This is sufficiently long to precisely expose the plate to the lasers manually by simply opening the zero aperture iris and leaving it open, but short enough to avoid possible perturbing vibrations. 
Because with the second plate/object support (PO2) the objects are not directly in contact with the plate, it should be used in quieter environments. This support is specifically suited to small groups. It is designed for single-shot reflection/transmission holograms using the diverging laser beam coming from the bottom (right side, figure 2). The holographic plate is vertically fixed onto the support with four screws and plastic washers. Two objects are placed on both sides of the holographic plate and are secured with small pieces of modeling clay. The one behind (elephant on figure 2) is used to create a reflection hologram while the one in front (cat) is used to create a transmission hologram. A small mirror can be added at the side to improve the brightness of the transmission hologram through better illumination of the object. Due to the divergence of the beam, this mirror is illuminated by a part of the reference beam and can be oriented so as to illuminate the object.

All the equipment needed to develop the holographic plates is inside the case: the developing products (developer, bleach and wetting agent), the corresponding tools (thermometer to control the temperature of the developer, syringe, measuring beaker, hair dryer to dry the plates after development) and a few $4 " \times 5 "$ silver halide Ultimate 08 color plates [11]. Note that these plates are not pre-swelled, to keep the rendering colors as real as possible. Developing an Ultimate plate takes approximately $15 \mathrm{~min}$ :

- dilute 1:10 in water developer (in darkness) : $6 \mathrm{~min}$ at $20^{\circ} \mathrm{C}$,

rinse (in darkness) in running water $1 \mathrm{~min}$,

bleach until transparent (3-4min),

rinse in running water $1 \mathrm{~min}$,

soak $1 \mathrm{~min}$ in water containing a few drops of wetting agent,

dry 2 min (with absorbent paper, then with a hair dryer).

\section{Plate/object anti-vibration support PO1: qualitative and quantitative proof of robustness}

The brightness of a hologram is defined by: (1) the intensity of the light diffracted by the hologram to reconstruct the image, which depends on the number of Bragg planes constructively reflecting the incoming light and (2) the spatial resolution $[12,13]$, which depends on the finest detail available in the interference pattern recorded in the silver halide emulsion of the holographic plate. Thus the size of the silver grains determines the definition of the reconstruction: the smaller the grain, the higher the definition. The grain size of the Ultimate holographic plates used for this device is extremely small, $8 \mathrm{~nm}$ on average [11].

In the following, the $z$ axis is perpendicular to the surface of the holographic plate. For a reflection hologram, the fringe planes, also named Bragg planes [2], are perpendicular to $z$ and spaced a distance $d$ apart. Ensuring sufficient intensity

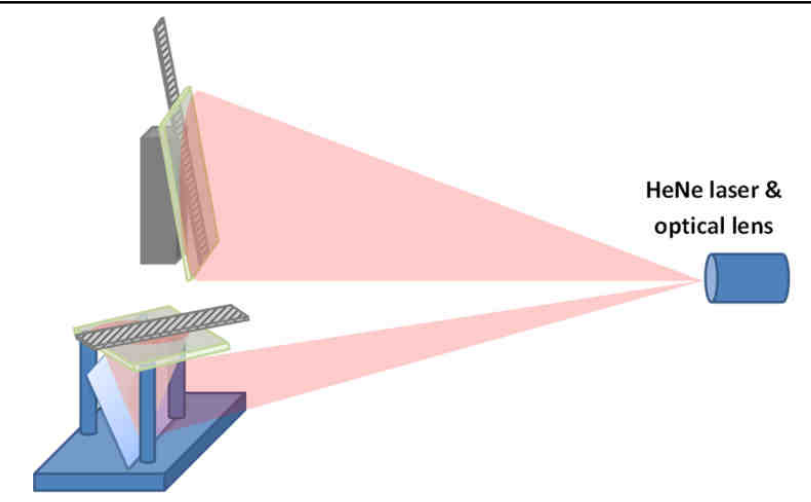

Figure 3: Experimental setup used to characterize vibrations - the laser beam simultaneously illuminates the PO1 support and a classic setup. A radio program is running during printing time at various noise levels: 40 , 60 or $70 \mathrm{~dB}_{\mathrm{A}}$.

for the reconstruction of the hologram requires that there be no vibrations along $z$ during recording. This is a limitation: the planes must not be blurred. For an angle $2 \alpha$ between the reference beam and the scattered object beam, a laser wavelength $\lambda$, we have:

$$
d=\frac{\lambda}{2 \sin \alpha}
$$

Here $\lambda=632.8 \mathrm{~nm}$ and $\alpha=90^{\circ}$, the Bragg planes are spaced about $d=315 \mathrm{~nm}$ apart in the medium. This cannot be easily seen with an optical microscope.

However in the $(\mathrm{x}, \mathrm{y})$ plane parallel to the plate, the fringes at the object's edge can be of the largest sizes visible under an optical microscope: they follow the Fresnel diffraction pattern [10,14]. Therefore, in the case of a reflection hologram of a point-like object, the constructive fringes get closer to the fringe order $n$ (zero order is at the edge of the object). In this case, fringe spacing $i_{n}$ evolves as: $i_{n}=\sqrt{\frac{\lambda D}{2 n}}$ for $n>>1$, with $D$ the object-plate distance. Fringe intensity is also decreasing with the increase of order $n$. If the object is not like a point, the interference pattern is more complicated. But fringe spacing always decreases with the increase of $n$. Moreover, if the object is placed at $\mathrm{y}=0$, fringe spacing is decreasing with $|y|$. Thus, the smallest recorded fringe spacing will indicate the smallest detail visible in the reconstruction. If the object has a simple shape, like the edge of a metal ruler, the smallest detail is easier to measure.

When there is noise during plate exposure to the laser light, an optical microscopy view of the fringe plane can provide information on loss of spatial resolution. Note that loss of image intensity is harder to measure because optical microscopy only provides information on the $(\mathrm{x}, \mathrm{y})$ vibrations, along the plane of the hologram.

Here, we tested the effectiveness of the PO1 support by simulating a public exhibition. Two holograms of the same object (a metal ruler) were realized at the same time: one using PO1 and the other not using PO1 but placing the ruler directly on the plate (see figure 3 ). To reproduce a public exhibition environment, a radio program was running in the room: the experiment was repeated under various noise 
conditions (measured in human ear weighted units, $\mathrm{dB}_{\mathrm{A}}$, at the recording site): 40,60 and $70 \mathrm{~dB}_{\mathrm{A}}$.

Pictures of the holograms obtained under these conditions are shown in figure 4 . Note that the hologram no longer appears in the $70 \mathrm{~dB}_{\mathrm{A}}$ noisy condition. Only a small part close to the mechanical contact point is seen. This is qualitative proof of the robustness of PO1.

Microscopy measurements provide more quantitative insights. Micrographs of the plane (x,y) were obtained with a metallographic optical microscope along the edge of the ruler (figure 5). These micrographs were taken in the white circles plotted on the hologram pictures from figure 4. A $90^{\circ}$ rotation was performed with respect to the picture in order to plot the gray level profiles of figure 5 in the same direction. The gray level profile is a signature of the recorded light intensity and is encoded on 8bit. When PO1 is used, the intensity level of the fringes under the $40 \mathrm{~dB}_{\mathrm{A}}$ (no noise) and the $70 \mathrm{~dB}_{\mathrm{A}}$ (noisy) conditions are similar. The intensity level is decreasing from the edge of the ruler to the external part that is clearly visible in the no-noise condition or using PO1: if the object edge is in $y=0$, the intensity is decreasing with $|y|$.

Variations in intensity were measured with the interferometric visibility defined as $\Gamma=\frac{I_{\max }-I_{\min }}{I_{\max }+I_{\min }}$. They correspond to the intensity modulation perpendicular to the edge of the object. Results on resolution and interferometric visibility with the gray level profile are reported in Table 1.

\begin{tabular}{|c|c|c|c|c|}
\hline & \multicolumn{2}{|c|}{} & & PO1 \\
\hline Noise $\left(\mathrm{dB}_{\mathrm{A}}\right)$ & 40 & 60 & 70 & 70 \\
\hline $\begin{array}{c}\text { Interferometric } \\
\text { visibility } \\
\Gamma\end{array}$ & 0.65 & 0.41 & 0.19 & 0.91 \\
\hline $\begin{array}{c}\text { Smallest detail } \\
\varepsilon( \pm 0.5 \mu \mathrm{m})\end{array}$ & 1.4 & 1.5 & 2.5 & 0.8 \\
\hline
\end{tabular}

Table 1: Resolution and interferometric visibility obtained under the different noise conditions

The interferometric visibility is much better without noise or with PO1. Under very noisy conditions $\left(70 \mathrm{~dB}_{\mathrm{A}}\right)$, the smallest detail visible in the plane is three times better with PO1.

Holograms realized with PO1 were roughly identical whatever the noise conditions. These results confirm that recording using the weight of the object on the plate directly to reduce the effect of vibrations is an effective and relevant method of producing holograms under noisy conditions.

Sound vibrations correspond to the mechanical resonance of glass, the material of the plate. If the object vibrates together with the glass, then relative oscillations are damped. The shorter the mechanical loop, the smaller the oscillations between the plate and the object. Therefore, the small circle in the $70 \mathrm{~dB}_{\mathrm{A}}$ condition without support shown in figure 4 corresponds to the lower part of the ruler where the table adds a contact between the ruler and the plate. The signal is quickly lost when the mechanical loop increases $(5 \mathrm{~mm}$ away from the table). This suggests that the best way to ensure optimum reproducibility when making a hologram is to use the full weight of the object, and thus to place the plate horizontally.

We did not test the PO2 support since this is equivalent to using the mount without support. Mechanical loop is long

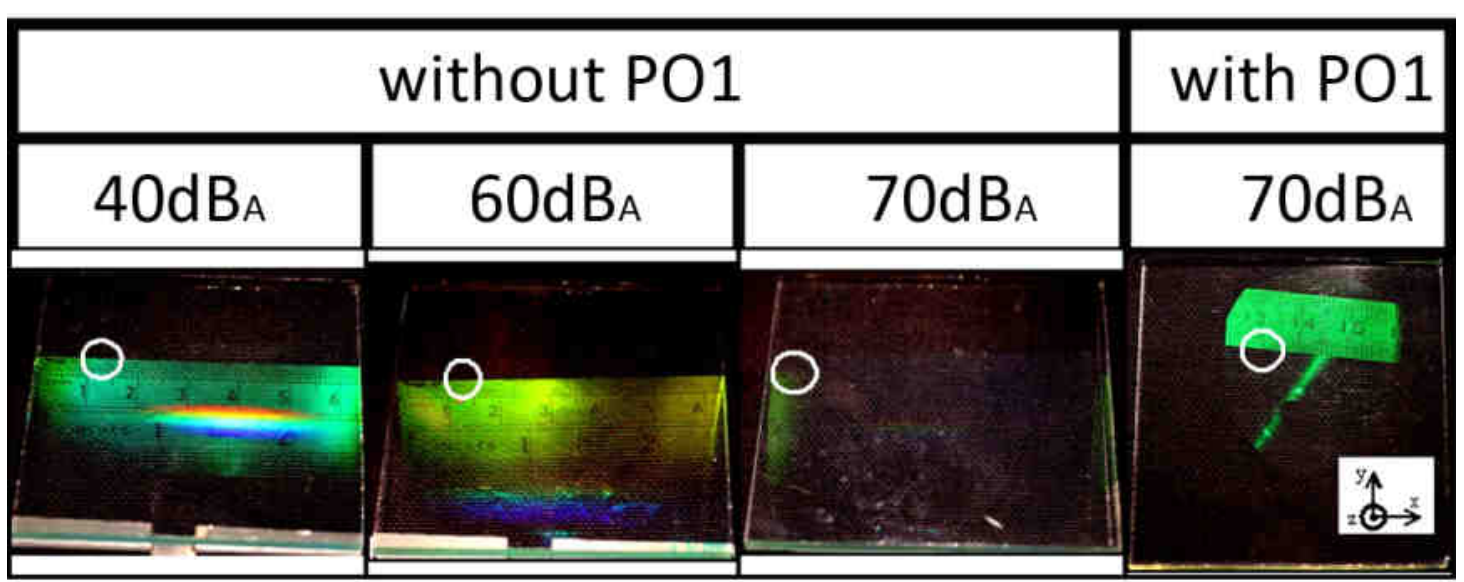

Figure 4: Pictures of the holograms obtained for three controlled noise conditions with and without PO1. Even under the worst acoustic conditions, it is the PO1 support that enables the best hologram to be obtained.

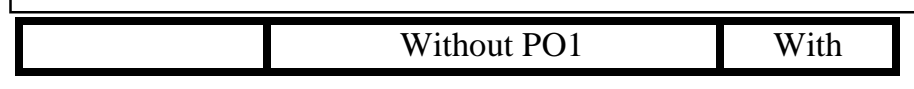

because the object and the plate are placed vertically. PO2 is only intended for use under quieter environments. 


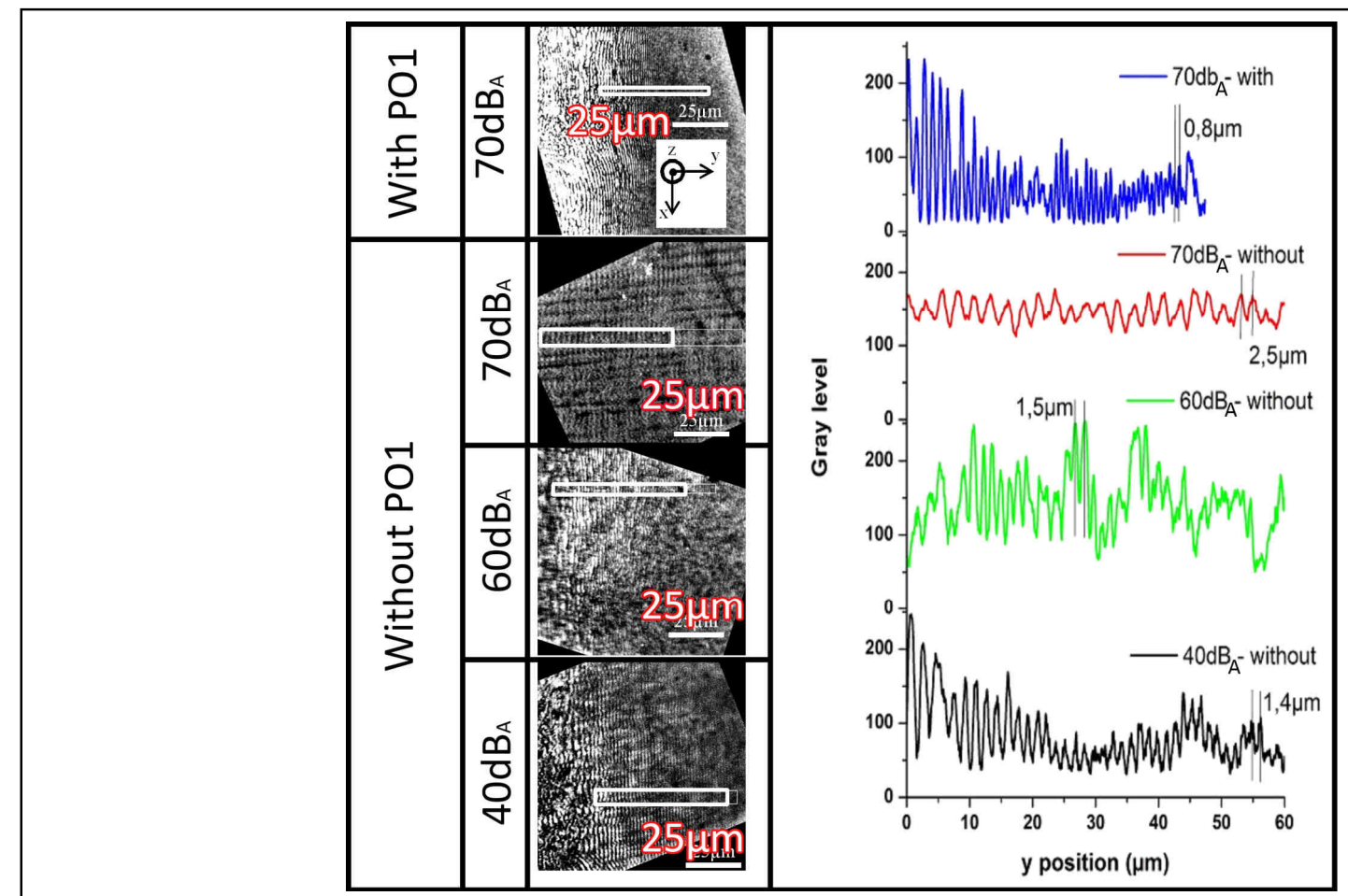

Figure 5: Micrographs taken in the white circles from pictures in figure 4 show the recorded fringes. The gray level profiles plotted on the right are extracted from the white rectangular zones. The interferometric visibility is clearly better using PO1. The size of the smallest visible detail is measured on the plane of the recording medium.

\section{Experiments for popular science and pedagogy}

The holography color device can be used to realize multiple experiments for outreach purposes, such as the color Denisyuk reflection holograms described below or for high level physics courses. For example, the holography color device was used for experimental projects with small groups of Master students at Aix-Marseille University. During 6 3hours sessions, they worked on the realization and the characterization of diffraction gratings. They began by creating a single-shot transmission/reflection hologram (with plate/object support PO2) to understand the differences between them. Once developed, the distinguishing feature of such a hologram is that it contains in the same volume a reflection and a transmission hologram. Depending on how it is observed, with laser by transmission or with a white light by reflection [15], students can see each of the two holograms. This experiment has great pedagogic value: it raises the student's awareness of the effect of the relative orientation of the object/reference beams with respect to the plate. They also master the different phases of realization of a hologram: setup mounting, exposure time calculation, and development. The students subsequently created and characterized several diffraction gratings and mounted a complete transmission spectrometer [16].

The reader will find descriptions of different holography projects using the holography color device in Additional Information.

\subsection{Color Denisyuk reflection holograms}

For science outreach purposes, color reflection holograms are spectacular $[17,18]$.

The main advantage of the device is to allow the realization of spectacular 4" 5 " Denisyuk color holograms [19]. For perfect reproducibility of a wide range of color shades, at least three lasers with wavelengths corresponding to three independent primary colors need to be used [20]. However, the realism of «color » holograms realized using only red and green lasers is very impressive too, even though it is not possible to obtain pure white and purple/blue shades (figure 6). When created during science outreach events, the impact on the audience of such «two-color» $4 " \times 5 "$ holograms is very strong, much greater than the impact of small monochromatic holograms. Adding an expensive third laser in the blue region does not therefore appear essential: the green laser gives the hologram its luminosity, while combining it with the red laser gives a wide range of color shades. Holograms produced with red and green lasers represent a good compromise between cost and audience impact. As we will see later in the text, the use of the two $\mathrm{red} /$ green lasers also considerably widens the scope for experiments illustrating the principles of holography. The redesigned PO1 releases the experimentalist from concern over unwanted disturbing vibrations. The device has been successfully used to realize holograms in noisy environments 
such as an amphitheater full of students or during outreach sessions in a museum and in the street with a dark tent.

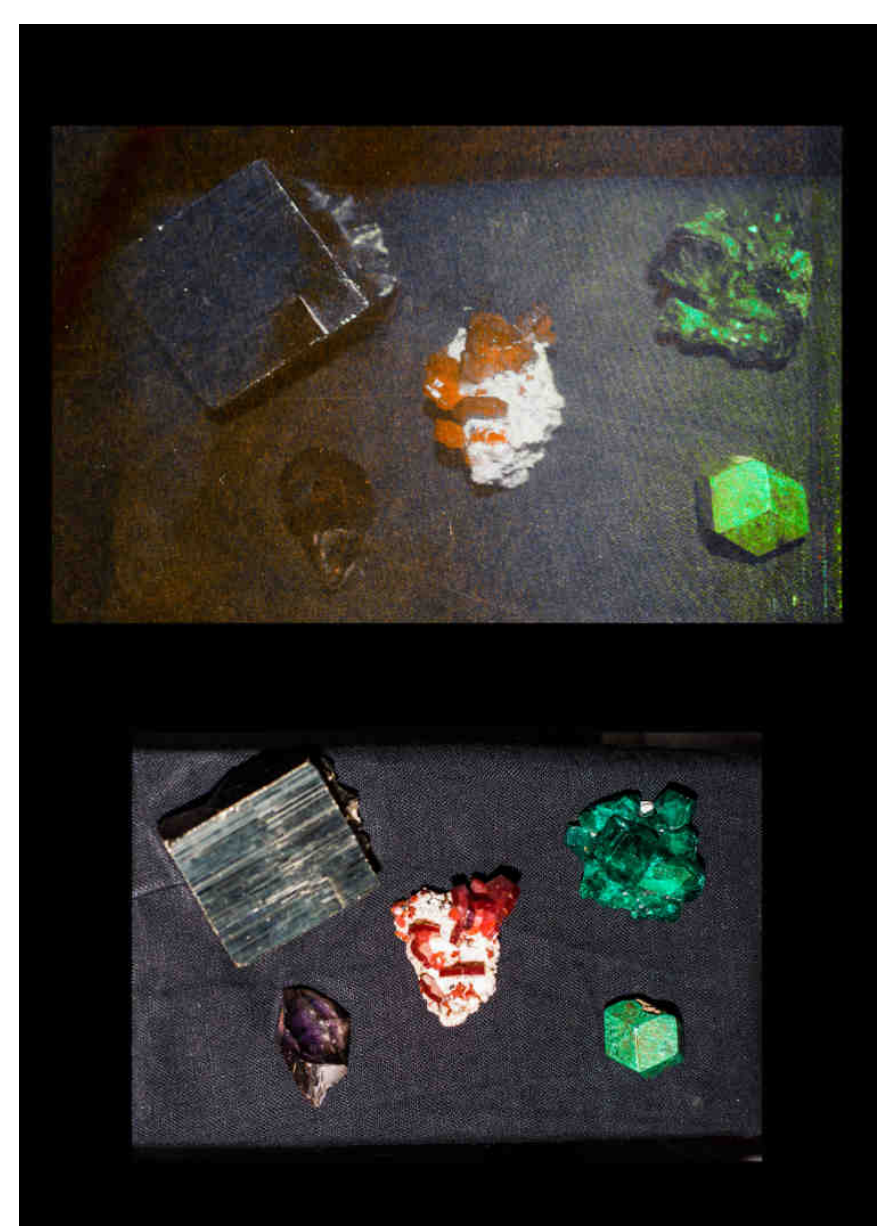

Figure 6: Two-color (red/green) hologram of crystals (up) and originals (down). Crystals are, from the top left to the bottom right of the image: gold-yellow pyrite, deep green dioptase, red vanadinite, dual-pyramid purple quartz, and pale green malachite.

\subsection{Holographic diffraction gratings}

Holographic diffraction gratings are widely used in spectroscopy for research and industrial spectroscopic applications. Such an optical element is obtained when two coherent equally polarized monochromatic optical beams of equal intensity intersect each other onto a photo-sensitive plate. A diffraction grating can also be created from a single collimated coherent beam, if it is reflected back upon itself. The use of PO1 allows this configuration: the mirror is placed vertically against the rear post, with the holographic plate placed at the original mirror position (figure 7) [21]. A standing wave pattern will be formed, with intensity maxima forming planes parallel to the wavefronts. Its intersection with a photo-sensitive medium yields on its surface a sinusoidal varying intensity pattern, whose spacing $i$ depends on angle $\theta$ as defined in figure 7. For a collimated beam and a laser wavelength $\lambda$, we have:

$$
i=\frac{\lambda}{2 \sin \theta}
$$

The number of grooves per millimeter can be controlled through the choice of laser wavelength $\lambda$ and angle $\theta$. For pedagogic use of color holography, it is possible to obtain values of up to $2800 \mathrm{~mm}^{-1}$ on a 4 " $\times 5$ " holographic plate.

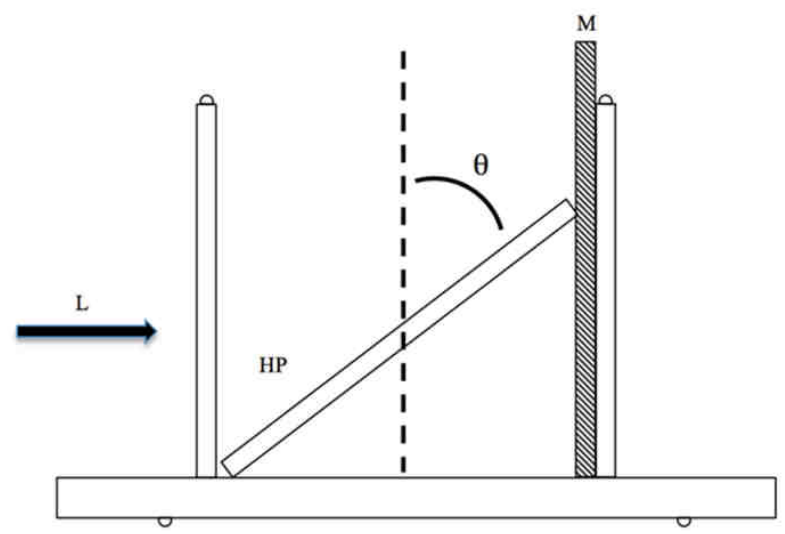

Figure 7: Experimental setup for the realization of a holographic diffraction grating with PO1. The laser beam L is coming from the left side. The holographic plate HP is leaning against the mirror $\mathrm{M}$, emulsion on the mirror side. The inclination of HP can be varied by changing the position of the rear post.

Once the diffraction grating is realized, the number of grooves per millimeter $a_{\text {exp }}$ can be experimentally obtained by measuring the angle of diffraction $\beta_{2}$ of an incident collimated laser beam with an incident angle $\beta_{1}$ with respect to the normal [22]:

$$
\sin \left(\beta_{1}\right)+\sin \left(\beta_{2}\right)=\frac{\lambda}{i_{\text {exp }}}=\lambda \cdot a_{\exp }
$$

Note that if $\beta_{1}=\theta$, the measured angle of diffraction $\beta_{2}=\theta$ also with respect to eq. (2). A diffraction grating was realized using the green laser and with the mirror inclined at an angle of $\theta=41.5^{\circ}$. The use of relation (3) with two lasers at $671 \mathrm{~nm}$ and $473 \mathrm{~nm}$ gives $a_{\text {exp }}=2513 \mathrm{~mm}^{-1}$ and $2457 \mathrm{~mm}^{-1}$, respectively. This is in very good agreement with the value predicted from equation (2): $\mathrm{a}_{\text {th }}=$ $2491 \mathrm{~mm}^{-1}$. The effect of the wavelength on the resulting value of $i$ can also be explored. Two diffraction gratings were successively created with the red and green lasers of the device. The use of relation (3) gave $a_{\exp 632}=2254 \mathrm{~mm}^{-1}$ and $a_{\exp 532}=2714 \mathrm{~mm}^{-1}$. As expected, the ratio $a_{\text {exp } 532} / a_{\text {exp } 632 n m}=1.19$ was equal to the ratio of the laser wavelengths $\lambda_{\text {red }} / \lambda_{\text {green }}$. These two gratings can be realized at the same time on the same plate, so that two holographic gratings can coexist with the corresponding resolutions. 
Note that when realizing diffraction gratings with the device, the laser beam is divergent. Thus, the optical wavefront is spherical and relation (2) is not strictly valid. This means that the distance between successive fringe patterns 1) differs from the value for a collimated beam and 2) is not constant over the diffraction grating surface. To check how important these effects are, we measured the variation of the number of grooves per millimeter over the surface of a diffraction grating realized with the device: $\Delta a / a<4 \%$. Moreover, we realized a diffraction grating with a collimated beam by adding a second converging lens after that used to expand the laser. The ensuing variation was $2.2 \%$ lower than that of the diverging beam configuration. It can therefore be concluded that relation (2) is a good approximation in our case. An explanation for these results is that PO1 was positioned far from the laser sources, at a distance of $L \approx 4.5 \mathrm{~m}$, so that the incoming beam is only slightly spherical.

\section{Conclusion}

Our first monochromatic pedagogic tool for holography developed in 2012 has been widely used for teaching purposes at Aix-Marseille University, for continuing education and for popular science events. Since 2015, it has been upgraded to a holography color device described in this publication. This provides more pedagogic opportunities to engage an audience, with no particular need for an expensive active or passive anti-vibration apparatus. The device is portable and contains a stable dual (red/green) beam diverging light source (the case), an anti-vibration plate/object support, a single shot reflexion/transmission support and the developing chemistry.

To test the effectiveness of the anti-vibration support in controlling vibrations, we used the Fresnel diffraction recording of a metal ruler. This allowed us to measure the smallest detail available on the recording, used to indicate the hologram's brightness. Several noise conditions were tested and unambiguously showed that noise is not a problem when using the anti-vibration support. The device can be used for outreach purposes, during public exhibitions, science festivals, conferences, and for middle and secondary school in-service teacher training.

Associated with an additional support included in the holography color device, it is possible to develop more complex educational experiments compare to the first pedagogic tool: color holography, comparison of reflection/transmission holograms, Bragg effect, angular and wavelength multiplexing principles, diffraction gratings with control of the number of grooves per millimeter, notch filters with central wavelength control.

Holography offers opportunities to explore a wide range of fundamental principles of optics. At the same time, the spectacular ability of holography to reproduce reality is an excellent source of motivation for students, as well as a very effective way of capturing the attention of any audience.

\section{Acknowledgements}

The authors would like to thank Yves Gentet for helpful discussions, Alain Baronnet for his collection of crystals and Marjorie Sweetko for improving the English. This work was supported by the French government's facility "Science à l'école", as part of the LUNAP operation.

\section{Additional information}

The holography color device could also be used to illustrate data storage (Holographic Versatile Disc) with wavelength and angle multiplexing. For angle multiplexing, PO2 is simply rotated to change the incident angle of the laser beam between two laser exposures of two different objects on the same plate. Once developed, the image of one object or the other can be seen, depending on the angle of observation of the hologram. This experiment can be performed with a transmission or a reflection hologram, depending on the position of the object: in front of or behind the plate. The wavelength multiplexing effect can be realized with PO1: the same holographic plate is successively exposed to the two lasers, and the object is changed between the shots. Once developed, the two red and green images are superimposed when the hologram is illuminated with white light. When it is exposed to red and green lights, the corresponding image can be seen, thus illustrating the wavelength selectivity of the hologram.

Moreover, as with the pedagogic tool for monochromatic holography, it is possible to realize experiments on doubleexposure holographic interferometry (mechanical stress diagnostics) and notch filters (Raman spectroscopy). For more information on these experiments, the reader can refer to our previous publication [5]. The experiments are enriched by the presence of two lasers in the holography color device. For example, a double-notch filter can be realized by using a front surface mirror exposed to the red and green lasers at the same time, with two reflection spectral bands centered on the lasers' wavelengths.

\section{References}

[1] Jeong T.H. 2013 J. Physics: conf. series 415012062

[2] Saxby G and Zacharovas S 2015 Practical holography (4th edition, CRC Press, New York)

[3] Jeong T.H. 2000 Optics \& Photonics News 1114

[4] Jeong T.H. 1975 Am. J. Phys. 43714

[5] Voslion Th and Escarguel A 2012 Eur. J. Phys. 331803

[6] Voslion Th, Escarguel A 2012 J. Phys.: Conf. Ser. 415012001 
[7] Escarguel A 2015 proceedings of the $10^{\text {th }}$ International Symposium on Display Holography,

http://holowiki.org/w/images/7/70/ISDH2015Escarguel.pdf

[8] Windle et al. 1999 Gait \& Posture 931

[9] Denisyuk Y. N. 1992 Leonardo 25425

[10] Gabor D 1948 Nature 161777

[11] http://www.ultimate-holography.com/

[12] Latychevskaia T, Longchamp J.N, Fink H.W 2012 opt.

Express 2028882

[13] Salançon E, Degiovanni A, Lapena L and Morin R 2018 Rev.

Sci. Instrum. 89043301

[14] Morin R 1994 Microsc. Microanal. Microstruct. 5501

[15] Caulfield H. J 1979 Handbook of optical holography,

(Academic Press, New York)

[16] Bell R. E 2004 Rev. Sci. Instrum. 754158

[17] Hubel P.M and Solymar L 1991 Appl. Optics 304190

[18] Hubel P.M, Ward A.A 1989 proc. SPIE 105118

[19] Denisyuk Y. N 1962 Akad. Nauk SSR 1441275

[20] Bazargan K 2013 J. Phys.: Conf. Ser. 415012028

[21] Sheridon N. K 1968 Appl. Phys. Lett. 12316

[22] James J 2007 Spectrograph design fundamentals (Cambridge University Press, Cambridge) 